EPJ manuscript No.

(will be inserted by the editor)

\title{
Hypernuclear Spectroscopy in JLab's Hall A
}

John J. LeRose ${ }^{1}$, A. Acha ${ }^{2}$, P.Bydžovský ${ }^{3}$, C.C. Chang ${ }^{4}$, E.Cisbani ${ }^{5}$, F. Cusanno ${ }^{5}$, C.W. de Jager ${ }^{1}$, R. De Leo ${ }^{6}$, R.J. Feuerbach $^{1,9}$, S. Frullani ${ }^{5}$, F. Garibaldi ${ }^{5}$, D.W.Higinbotham ${ }^{1}$, M. Iodice ${ }^{7}$, L. Lagamba ${ }^{6}$, P. Markowitz ${ }^{2}$, S. Marrone ${ }^{6}$, B. Reitz ${ }^{1}$, M. Sotona ${ }^{3}$, G.M. Urciuoli ${ }^{8}$, and The Hall A Collaboration

1 Thomas Jefferson National Accelerator Facility (JLab), Newport News, VA 23606, USA

2 Florida International University, Miami, FL 32306, USA

3 Nuclear Physics Institute, Rež, Czech Republic

4 University of Maryland, College Park MD, USA

5 INFN Roma, gr. Sanita' and ISS, I-00161, Rome, Italy

6 INFN Bari and Dipartimento di Fisica and Istituto Nazionale di Fisica Nucleare, Bari, Italy

7 INFN Roma Tre, Rome, Italy

8 INFN Roma, Rome, Italy

9 present address The College of William and Mary, Williamsburg VA 23187, USA

Received: date / Revised version: date

\begin{abstract}
Results are presented from a new experiment (E94-107) in Hall A of the Thomas Jefferson National Accelerator Facility (JLab) producing ${ }_{\Lambda}^{12} \mathrm{~B}$ using electroproduction, $\left(\mathrm{e}, \mathrm{e}^{\prime} \mathrm{K}^{+}\right)$. In the hypernuclear missing-mass spectrum the experiment achieves very good energy resolution (640 keV FWHM) by exploiting the characteristics of the High Resolution spectrometer pair and the exceptional beam quality available at JLab. The spectrometers were used with the addition an INFN provided pair of septum magnets to reach the desired small angles. Also, the Hall A standard complement of equipment was further augmented by the addition of a Ring Imaging Cherenkov detector (RICH) to achieve the best possible kaon identification.

PACS. 21.80.+a hypernuclei - 25.30.Rw Electroproduction reactions -21.60. Cs Shell model $-24.50 .+\mathrm{g}$ Direct reactions - 29.40.Ka Cherenkov detectors - 29.30.Aj Charged-particle spectrometers: electric and magnetic
\end{abstract}

\section{Introduction}

The advantages of using the $\left(\mathrm{e}, \mathrm{e}^{\prime} \mathrm{K}^{+}\right)$reaction to create and study hypernuclei include:

- high intensity and excellent energy resolution of the electron beam make it possible to identify hypernuclear levels with a resolution of the order of a few hundred $\mathrm{keV}$;

- photo- and electro-production reactions are characterized by large momentum transfer $(q \geq 250 \mathrm{MeV} / \mathrm{c})$ and strong spin-flip terms even at zero kaon production angles. This means that photons (real or virtual) can create hypernuclei in both natural and unnatural parity, low-spin, and high-spin states including those with deeply-bound $\Lambda$ hyperons;

- in contrast to $\left(\mathrm{K}^{-}, \pi^{-}\right)$or $\left(\pi^{+}, \mathrm{K}^{+}\right)$, electromagnetic production of the $\mathrm{K}^{+} \Lambda$ pair takes place on the proton which allows the study of hypernuclei not available otherwise $\left({ }_{\Lambda}^{12} \mathrm{~B}\right)$ including hypernuclei with large excess of neutral baryons $\left({ }_{A}^{7} \mathrm{He},{ }_{A}^{9} \mathrm{Li}\right)$. Comparison of the spectra of mirror hypernuclei, e.g. ${ }_{\Lambda}^{12} \mathrm{~B}-{ }_{\Lambda}^{12} \mathrm{C}$, can then shed light on the charge asymmetry of the hyperonnucleon interaction.
JLab, provides a unique opportunity to study hypernuclear spectroscopy using the heretofore little used $\left(\mathrm{e}, \mathrm{e}^{\prime} \mathrm{K}^{+}\right)$ reaction to produce the hypernuclear states. The first electroproduction experiments done in JLab's Hall C demonstrated the feasibility of such experiments [1].

\section{Experimental Equipment}

In order to effectively do high-resolution hypernuclear spectroscopy experiments with electron beams three ingredients are required. They are:

1. A high quality, high current, high duty factor, $\sim 4 \mathrm{GeV}$ electron beam

2. Two high resolution spectrometer arms

3. Excellent particle identification

\subsection{Electron Beam Requirements}

E94-107 required a continuous wave, $3.66 \mathrm{GeV}, 100 \mu \mathrm{A}$ electron beam with very small energy spread and vertical spotsize (energy spread $\sigma \leq 3 \times 10^{-5}$, spot size $\sigma \leq 100 \mu \mathrm{m}$ ). 
John J. LeRose et al.: Hypernuclear Spectroscopy in JLab's Hall A

With some effort the Accelerator staff were able to achieve these requirements. The beam spot size was measured using standard harp scan techniques. The energy spread was monitored continuously using a Synchrotron Light Interferometer (SLI) [2] to monitor the physical size of the beam at a point in the Hall $\mathrm{A}$ arc with large dispersion.

\subsection{High Resolution Spectrometers}

The standard equipment high-resolution spectrometer pair (HRS)[3] in Hall A was designed to deliver the required momentum resolution. However, the minimum angles with repect to the beamline of $12.5^{\circ}$ were too large. This shortcoming was alleviated by the introduction of a pair of superconducting septum magnets, each of which providing a $6.5^{\circ}$ horizontal bend. By moving the target postion $80 \mathrm{~cm}$ upstream and inserting the septum magnets on either side of the beamline the HRS pair at $12.5^{\circ}$ on either side of the beamline was able to detect kaons and electrons at $6^{\circ}$. Funding for the septum magnets was provided by INFN.

Due to their small bend angle and relatively short length $(80 \mathrm{~cm})$ the septum magnets made only a small perturbation on the standard HRS optics that was easily corrected by a small modification of the settings of the three quadrupoles in each arm. The quality and exact character of the optics transformation tensor were measured with a series of elastic scattering measurements using a $2 \mathrm{GeV}$ electron beam on $\mathrm{C}$ and Ta targets. Along with the elastic scattering, measurements were made using a sieve-like mask in front of each spectrometer to optimize and calibrate the angular reconstruction. The results of the calibration and optimization effort are illustrated in Fig. 1.

\subsection{Particle Identification}

In the electron arm the Gas Cherenkov counter gives pion rejection ratios up to $10^{3}$. The dominant background (knockon electrons) is reduced by a further two orders of magnitude by using the lead-glass shower and pre-shower counters, giving a total pion rejection ratio of $10^{5}$.

The identification of kaons detected in the hadron arm together with a huge background of protons and pions is one of the major challenges of the experiment. To reduce the background level in produced spectra, a very efficient PID system is necessary for unambiguous kaon identification.

The PID system in the hadron arm is composed of: a high-resolution scintillator plane, two aerogel threshold Cherenkov counters A1 and A2 [3,4] $\left(\mathrm{n}_{1}=1.015, \mathrm{n}_{2}=\right.$ 1.055) which were included in the trigger, and the RICH detector for offline analysis.

In the aerogel counters, charged pions (protons) with momenta around $2 \mathrm{GeV} / \mathrm{c}$ are above (below) the Cherenkov light emission threshold. Kaons emit Cherenkov light only in the aerogel with the higher index of refraction. Hence,

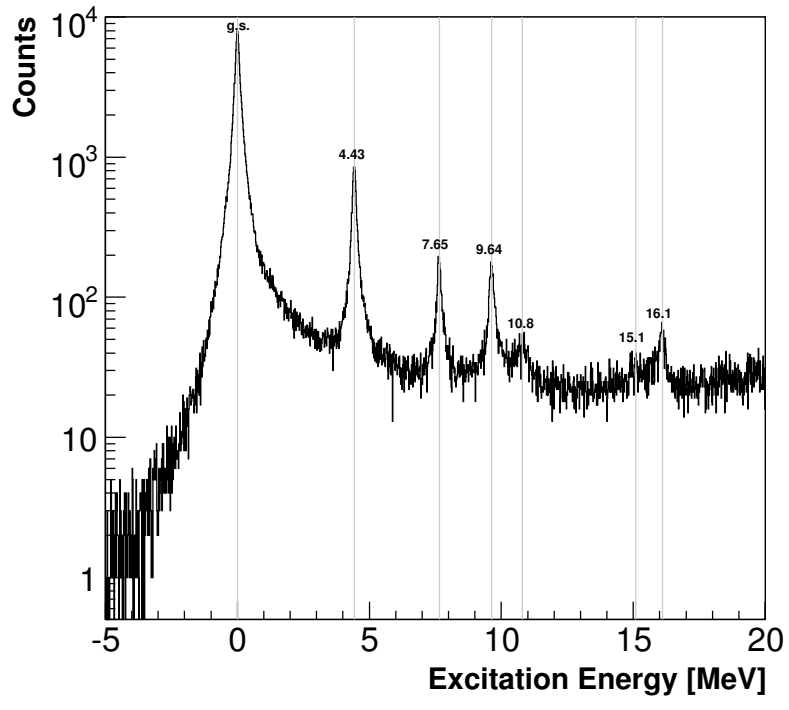

Fig. 1. Optimized elastic and inelastic electron scattering spectrum from ${ }^{12} \mathrm{C}\left(100 \mathrm{mg} / \mathrm{cm}^{2}\right)$ at $2 \mathrm{GeV}$. FWHM of all peaks is $\delta \mathrm{p} / \mathrm{p} \leq 1 \times 10^{-4}$.
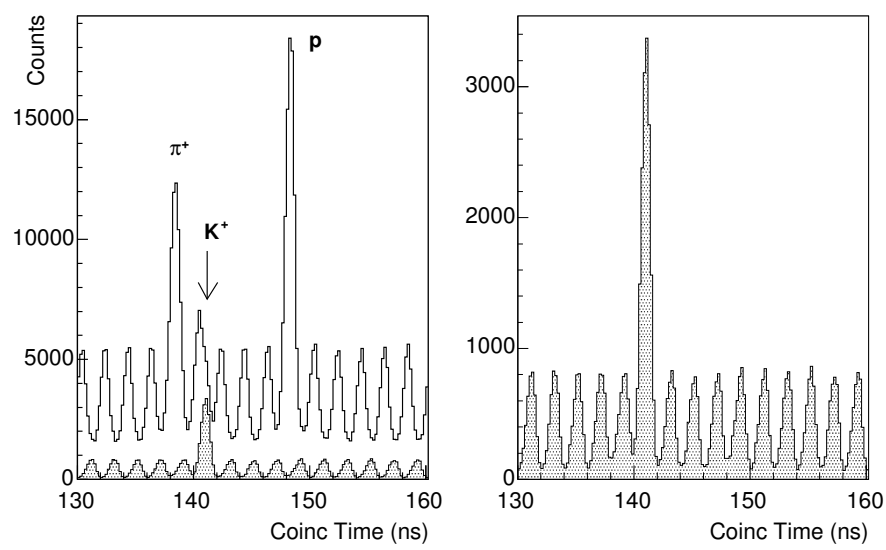

Fig. 2. Left Panel open plot: coincidence timing spectrum with only aerogel PID. Right panel and left panel filled plot: the same coincidence timing spectrum with RICH PID included. Only kaons remain.

the combination of the signals from the two counters distinguishes among the three species of hadrons.

The RICH operated successfully during E94-107 providing very satisfactory pion/kaon rejection at $2 \mathrm{GeV} / \mathrm{c}$, better than 1:1000 (corresponding to a pion/kaon angle separation $\geq 5 \sigma)$. Figure 2 illustrates the efficacy of the $\mathrm{RICH}$ in eliminating pions and protons from the detected particles.

The layout of the RICH is conceptually identical to the ALICE HMPID design [5]. It uses a proximity focusing geometry, a CsI photocathode, and a $15 \mathrm{~mm}$ thick liquid Freon radiator. A detailed description of the layout and the performance of the detector is given in $[6-8]$ as well as elsewhere in these proceedings. 

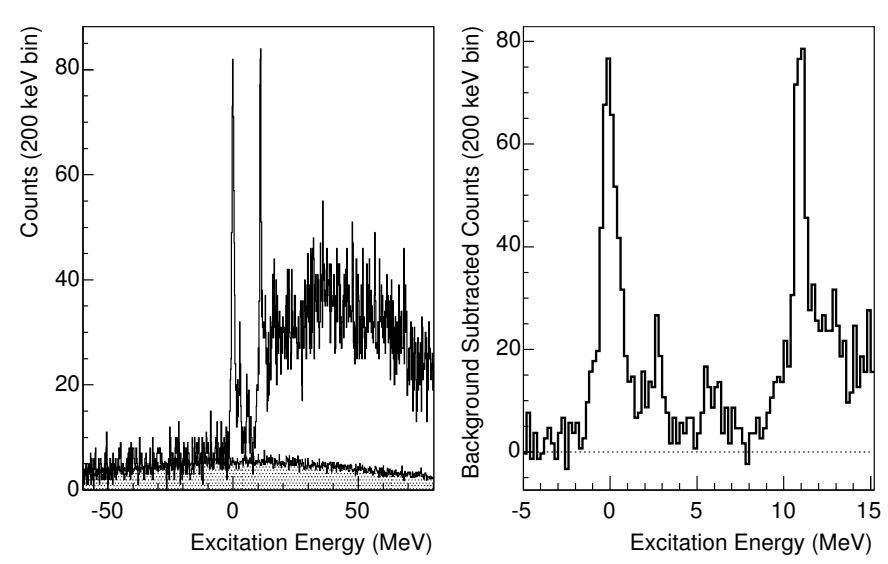

Fig. 3. Left panel: The open plot is the ${ }_{\Lambda}^{12} \mathrm{~B}$ excitation energy spectrum after kaon selection with aerogels and the RICH. The filled plot is a normalized accidental coincidence spectrum evaluated using a very large timing window. The right panel shows an exploded ${ }_{\Lambda}^{12} \mathrm{~B}$ spectrum after the background has been subtracted.

\section{Results for ${ }^{12} C\left(\mathbf{e}, \mathbf{e}^{\prime} \mathbf{K}^{+}\right){ }_{\Lambda}^{12} \mathbf{B}$}

The measured excitation energy spectrum of ${ }_{\Lambda}^{12} \mathrm{~B}$ from a $100 \mathrm{mg} / \mathrm{cm}^{2}$ target is shown in Fig. 3 with $200 \mathrm{keV}$ binning. Fig. 4 shows the six-fold differential cross section expressed in $\mathrm{nb} /\left(\mathrm{sr}^{2} \mathrm{GeV} \mathrm{MeV}\right)$. As discussed in the figure 3 caption, the background has been evaluated by fitting the data obtained for random coincidences in a large timing window and subtracted. No residual background in the negative range of $E_{x}$ is present after subtraction. Also shown in Fig. 4 is a fitted spectrum of six Gaussian peaks (solid line) with a polynomial for quasi-free breakup. The results of the fitting are reported in Table 1 . Note that the width of the fifth fitted peak at $10.95 \mathrm{MeV}$ is considerably narrower $(640 \mathrm{keV})$ than those of the other five $(>1 \mathrm{MeV})$. The larger widths are attributed to unresolved spin-doublet splitting or other unknown structures close to the expected states. The dashed line in Fig. 4 is the unnormalized result of a calculation.

The model that has been used to compare our data with the theory (dashed line in the figure) is obtained in the framework of the Distorted Wave Impulse Approximation (DWIA) [9] using the Saclay-Lyon (SLA) model [10] for the elementary $p\left(e, e^{\prime} K^{+}\right) \Lambda$ reaction. Shell-model wave functions for ${ }^{11} \mathrm{~B}$ and ${ }^{12} \mathrm{~B}$ were obtained using fitted $p$ shell interactions and a parametrization of the $\Lambda N$ interaction that fits the precise $\gamma$-ray spectra of ${ }_{\Lambda}^{7} \mathrm{Li}[11]$.

The very good agreement of the data with the predicted cross section is a first, though indirect, indication that the SLA model gives a good description of the elementary cross section at $Q^{2}$ as low as $0.07 \mathrm{GeV} / c^{2}$ and at forward kaon angles.

For the first time a measurable strength with good energy resolution has been observed in the core-excited part of the spectrum. The $s_{\Lambda}$ part of the spectrum is well reproduced by the theory. The distribution of strength within several $\mathrm{MeV}$ on either side of the strong $p_{\Lambda}$ peak

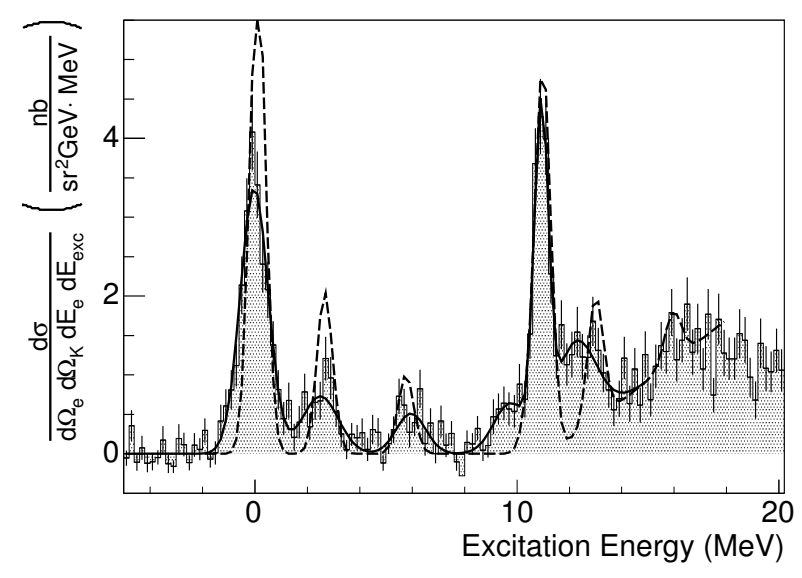

Fig. 4. ${ }_{\Lambda}^{12} \mathrm{~B}$ excitation-energy spectrum. The best fit (solid curve) and an unnormalized theoretical computation result (dashed curve) are superimposed on the data. See text for details.

at $10.95 \mathrm{MeV}$ should stimulate theoretical work to better understand the $p_{\Lambda}$ region.

\section{Conclusion}

Experiment E94-107 at JLab has completed a very succesful run. The addition of the septum magnets to the Hall A HRS's preserved the high quality optical properties designed in them and the RICH proved invaluable in cleanly identifying kaons. A good quality, high resolution, low background ${ }_{\Lambda}^{12} \mathrm{~B}$ spectrum has been obtained showing not only the dominant ground state $s-\Lambda$ and $p-\Lambda$ peaks but also measureable strength in the core excitation region between them. The $s_{\Lambda}$ part of the spectrum is well reproduced by the theory. The distribution of strength within several $\mathrm{MeV}$ on either side of the strong $p_{\Lambda}$ peak should stimulate theoretical work to better understand that region. Similar quality spectra on ${ }_{\Lambda}^{16} \mathrm{~N}$, and ${ }^{9}{ }_{\Lambda} \mathrm{Li}$ are being prepared

Analysis on ${ }_{\Lambda}^{16} \mathrm{~N}$, and ${ }^{9}{ }_{\Lambda} \mathrm{Li}$ is in a much more preliminary stage, but promises to provide spectra of similar quality to those presented here for ${ }_{1}^{12} \mathrm{~B}$. Preliminary results for those hypernuclei will be presented elsewhere in these proceedings.

This work was supported by the U.S. Department of Energy contract DE-AC05-84ER40150 Modification No. M175 under which the Southeastern Universities Research Association operates the Thomas Jefferson National Accelerator Facility, by the Italian Istituto Nazionale di Fisica Nucleare (INFN), by the U.S. Department of Energy under contracts W-31-109-ENG-38, DE-FG02-99ER41110, and DE-AC02-98-CH10886, and No.202/05/2142 of the Grant Agency of the Czech Republic.

\section{References}

1. T. Miyoshi et al., Phys. Rev. Lett. 90, 232502 (2003). 
Table 1. Levels and cross sections obtained by fitting the ${ }^{12} \mathrm{C}\left(e, e^{\prime} K^{+}\right){ }_{\Lambda}^{12} \mathrm{~B}$ spectrum.

Experimental data

\begin{tabular}{cccc}
\hline $\begin{array}{c}\text { Position } \\
(\mathrm{MeV})\end{array}$ & $\begin{array}{c}\text { Width }(\mathrm{FWHM}=2.37 \sigma) \\
(\mathrm{MeV})\end{array}$ & SNR & $\begin{array}{c}\text { Cross Section } \\
\left(\mathrm{nb} / \mathrm{sr}^{2} / \mathrm{GeV}\right)\end{array}$ \\
\hline $0.0 \pm 0.03$ & $1.25 \pm 0.07$ & 18.1 & $4.47 \pm 0.21($ st $) \pm 0.58($ sys $)$ \\
\hline $2.52 \pm 0.11$ & $1.62 \pm 0.33$ & 8.0 & $1.26 \pm 0.11($ st $) \pm 0.16($ sys $)$ \\
\hline $5.97 \pm 0.13$ & $1.29 \pm 0.31$ & 5.5 & $0.72 \pm 0.09($ st $) \pm 0.09($ sys $)$ \\
\hline $9.77 \pm 0.15$ & $1.53 \pm 0.38$ & 6.7 & $0.91 \pm 0.10($ st $) \pm 0.12($ sys $)$ \\
\hline $10.95 \pm 0.02$ & $0.64 \pm 0.05$ & 14.7 & $2.90 \pm 0.17($ st $) \pm 0.38($ sys $)$ \\
\hline $12.22 \pm 0.11$ & $1.90 \pm 0.24$ & 10.5 & $2.42 \pm 0.16(\text { st })_{-0.73}^{+0.41}($ sys $)$
\end{tabular}

2. Y-C. Chao et al., "Energy Spread Monitoring for the JLAB Experimental Program: Synchrotron Light Interferometers, Optical Transition Radition Monitors and Wire Scanners. Beam Instrumentation Workshop 2004,Ed. Thomas Shea, R. Coles Sibley III, AIP Conference Proceedings \#732.

3. J. Alcorn et al., Nucl. Instrum. Methods A 522, 294 (2004).

4. L. Lagamba et al., Nucl. Instrum. Methods A 471, 325 (2001).

5. CERN/LHCC 98-19, ALICE TDR 1, 14 August 1998.

6. M. Iodice et al., Nucl. Instrum. Methods A 553, 231 (2005).

7. F. Garibaldi et al., Nucl. Instrum. Methods A 502, 117 (2003).

8. F. Cusanno et al., Nucl. Instrum. Methods A 502, 251 (2003).

9. M. Sotona and S. Frullani, Prog. Theor. Phys. Suppl. 117, 151 (1994).

10. J.C. David, C. Fayard, G.-H. Lamot, B. Saghai, Phys. Rev. C 53, 2613 (1996); T. Mizutani, C. Fayard, G.-H. Lamot, B. Saghai, Phys. Rev. C 58, 75 (1998).

11. M. Ukai et al., Phys. Rev. C 73, 012501 (2006). 